\title{
LEIBNIZ Y LA HERMENEUTICA: PERSPECTIVISMO Y FUSION DE HORIZONTES ${ }^{1}$
}

\author{
Oscar M. Esquisabel \\ IECT-UNQ-CONICET-UNLP-UCA
}

\begin{abstract}
RESUMEN: El objetivo de este trabajo es exponer una aproximación a los puntos de contacto y de divergencia entre el pensamiento de Leibniz y la hermenéutica de Gadamer, en la perspectiva de justificar la actualidad del pensamiento leibniziano desde el punto de vista de la concepción actual de la racionalidad. La hermenéutica propone un concepto de razón “débil” que se funda en la noción de fusión de horizontes, que puede ser interpretado como una forma de perspectivismo. Como contrapartida, se examina el perspectivismo leibniziano desde el punto de vista de la armonía universal y del concepto de expresión como su fundamento. De este modo, la posibilidad de establecer correspondencias y transformaciones entre las diferentes perspectivas constituye un equivalente leibniziano la fusión de horizontes gadameriana. Además, gracias al concepto de "razón simbólica", se le reconoce la filosofía leibniziana cierta superioridad respecto de la hermenéutica gadameriana.
\end{abstract}

PALABRAS CLAVE: Leibniz; Gadamer; hermenéutica; racionalidad; perspectivismo.

\begin{abstract}
The goal of this paper is to present an approach to the convergences and divergences between Leibniz's thought and the gadamerian hermeneutics, as an essay to justify Leibniz's philosophy from the point of view of a contemporary view on rationality. Hermeneutics propose a "weak" concept of reason that is based in the notion of melting of horizons. Indeed, the melting of horizons can be interpreted as a form of perspectivism. In turn, Leibnizian perspectivism is examined from the point of view of the universal harmony and of the concept of expression as its ground. In this way, the possibility of establishing correspondences and transformations among different perspectives can be considered as a Leibnizian equivalent to the gadamerian melting of horizons. Moreover, through the notion of "symbolic reason", it must be recognized certain superioriy of Leibniz philosophy over gadamerian hermeneutics.
\end{abstract}

KEYWORDS: Leibniz; Gadamer; hermeneutics; rationality; perspectivism.

Hace más de cincuenta años que Gadamer publicó su obra fundamental, Verdad y método. ${ }^{2} \mathrm{Y}$ sin embargo, su obra dejó una huella indeleble en la filosofía del siglo XX, no tanto por ser una teoría filosófica, si es que hay

1 Este trabajo ha sido realizado en el marco del Proyecto de Investigación PIP 11220110100364 "Diagramas, visualizacióny formalismo. Problemas históricos y sistemáticos en la filosofía de las ciencias formales de Leibniz a Peirce y Hilbert".

2 Gadamer (1960). 


\section{Dossiê Leibniz, Dissertatio - Volume Suplementar 03 I UFPel [2016]}

rastro de ello en Verdad y método, sino más bien por haber contribuido a instaurar una forma de ver la racionalidad y la tarea filosófica que, de distintas maneras, se ha convertido en un patrimonio común de una gran variedad de corrientes filosóficas. Que hay que integrar la historia con el análisis conceptual, que toda forma de racionalidad se encuentra más o menos situada o condicionada por su contexto, que no debemos esperar obtener resultados filosóficos absolutamente últimos, que en la racionalidad humana el lenguaje cumple un papel fundamental y que hay que confiar en nuestra la racionalidad dialógica tanto en los aspectos teóricos como prácticos de la vida humana, son tesis de la hermenéutica que han adquirido un firme arraigo en la práctica filosófica de esta segunda mitad del siglo XX.

Sin embargo, nuestro intento de mostrar convergencias entre la hermenéutica y la filosofía leibniziana parece condenado al fracaso desde un primer momento. En efecto, la filosofía hermenéutica plantea la idea de una racionalidad débil o históricamente situada, en la que debemos o deberíamos despedirnos del ideal filosófico de una fundamentación y una verdad absolutas. Más aún, la hermenéutica se sitúa en una cierta oposición a la ciencia "objetificadora" o "cosificante". En particular, en la desconfianza de la hermenéutica hacia las ciencias, especialmente las ciencias de la naturaleza, se desenmascara el juicio negativo de Heidegger hacia el modo de pensar científico como manifestación de la razón calculante: las ciencias no piensan, sino que enmascaran la cosificación del mundo y del hombre. 
En este sentido, frente a la actitud hermenéutica, el pensamiento de Leibniz constituye una forma extrema de racionalismo que se expresa en el principio de razón suficiente: "nada es sin razón" o "nada es sin fundamento". El imperativo de fundamentación que expresa este principio se manifiesta, por una parte, en una metafísica de un ente absoluto o necesario, por una parte, y, por la otra, en la contraparte de una razón humana que aspira legítimamente a un conocimiento rigurosamente fundado, a partir del ideal de un cálculo lógico generalizado. Así, Leibniz sería un paso necesario hacia esa razón calculante de la que hablamos y que, a través de la informática, culmina en el nihilismo de imperio de la tecnociencia. En este camino de la desesperación, la monadología leibniziana se desenmascararía, finalmente, y a través del subjetivismo del idealismo alemán, como la mera pulsión vital que busca autoafirmarse como vida mediante la proyección de esquemas estabilizadores del caos, como una manifestación de la voluntad de poder. En este sentido, no podría haber mayor tensión entre la hermenéutica y la filosofía leibniziana, en la medida en que esta última sería una expresión más del destino "ontoteológico" de la metafísica occidental.

Ciertamente, no es mi intención realizar un balance de este relato heideggeriano que presenté aquí de manera muy simplificada y que forma parte del trasfondo conceptual, aunque no excluyente, de la hermenéutica gadameriana. Antes bien, trataré de proponer una visión de conjunto que muestre más bien las convergencias entre la hermenéutica y algunos aspectos 


\section{Dossiê Leibniz, Dissertatio - Volume Suplementar 03 I UFPel [2016]}

centrales de la filosofía leibniziana, en particular, a partir del concepto general de punto de vista o perspectiva. En efecto, tanto la hermenéutica como la filosofía leibniziana pueden entenderse como formas de perspectivismo, es decir, como formas de comprensión de nuestro acceso al mundo y a nosotros mismos que proponen el punto de vista y el aspecto con que se nos revela nuestro mundo como los ejes centrales de dicho acceso. En este sentido, los diversos aspectos del perspectivismo leibniziano que iré desgranando a continuación, nos proporcionarán un medio para comprender mejor los puntos de contacto entre la filosofía leibniziana y la hermenéutica de Gadamer. Al mismo tiempo, trataré de mostrar que cuota de leibnizianismo puede ayudar a completar algunas falencias del pensamiento hermenéutico que se revelan, precisamente, a la hora de pensar la dimensión formal de las cosas. Precisamente, frente a la crisis epocal de la razón "fuerte" o "dura", la hermenéutica pretende plantear un concepto de racionalidad que, sin perder la esperanza de alguna clase de fundamentación debilitada, deja lugar también para los aspectos históricos, contextuales y situacionales. No obstante, y a pesar de su crítica al subjetivismo, justamente por no haber podido escapar de las tentaciones del relato heideggeriano acerca de la historia de la metafísica, la hermenéutica gadameriana permanece adherida a algunas de las tesis centrales de dicho relato y es allí, precisamente, donde la filosofía leibniziana puede auxiliarnos para dar cuenta de esos aspectos que la hermenéutica ignora o, directamente, excluye. En efecto, si la hermenéutica aspira a ser una concepción 
universal de la racionalidad, no podrá serlo si no abarca todos los aspectos de la actividad y la creación humanas.

Por esa razón, mi propuesta no apunta a proponer una valoración historiográfica del pensamiento de Leibniz, aunque no excluiré algunas consideraciones en ese sentido, sino que, más bien, intenta ofrecer una reformulación de tesis leibnizianas que considero valiosas para la situación filosófica actual, considerada desde un punto de vista hermenéutico. Séame permitido esta nueva vuelta de tuerca leibniziana para presentar una visión crítica, pero positiva, de la hermenéutica.

\section{El perspectivismo hermenéutico}

La hermenéutica como posición filosófica no se limita solamente a ser una propuesta en relación con la comprensión y recepción de tradiciones, mucho menos pretende proporcionar una "metodología" para la interpretación de productos culturales, sino que sostiene, antes bien, que nuestro acceso al mundo en general es de carácter comprensivo e interpretativo. Así, Gadamer potencia y despliega la tesis heideggeriana de que la comprensión y la interpretación son nuestro modo de existir y relacionarnos con el mundo. Ahora bien, uno de los capítulos fundamentales de la hermenéutica es su concepción de que toda comprensión e interpretación tiene lugar en el marco de un horizonte de comprensión. Si bien Gadamer no caracteriza completamente el 


\section{Dossiê Leibniz, Dissertatio - Volume Suplementar 03 I UFPel [2016]}

concepto de horizonte de comprensión, constituye una pieza fundamental de su concepción hermenéutica.

Una aproximación a este concepto tan complejo y difícil de asir es concebirlo como la proyección de posibilidades interpretativas sobre aquello que se pretende comprender o interpretar. Ciertamente, Estas posibilidades interpretativas no son puras creaciones libres y subjetivas del agente comprensor, sino que le vienen dadas por su situación histórica, por el conjunto de categorías y creencias compartidas y, especialmente, por el lenguaje, entendido como el reservorio o fondo de significados compartidos. Así, el lenguaje mismo es una "perspectiva del mundo" (Weltansicht), por lo que el horizonte de comprensión e intepretación es fundamentalmente de naturaleza lingüística. ${ }^{3}$

Nunca se enfatizará lo suficiente el hecho de que el horizonte, entendido de esta manera, no es solamente una proyección de la vida histórica de una cierta comunidad social o cultural o, utilizando conceptos de otra procedencia, una "mera forma de vida", sino que es, al mismo tiempo, una condición bajo la cual se nos presenta el mundo en cuanto tal y, por esta razón, aspira a una cierta "verdad" o, si se quiere atenuar un concepto tan exigente, a una cierta "legitimidad", por lo que en este sentido, un horizonte de comprensión y de interpretación se presenta siempre con la pretensión de que se lo reconozca como legítimo, es decir, tiene una "pretensión de verdad", que,

\footnotetext{
${ }^{3}$ Gadamer (1960), p.446 [531]. Las referencias entre corchetes remiten a la trad. esp.
} 
por supuesto, no necesariamente tiene que coincidir con lo que se puede tematizar explícitamente en él. ${ }^{4}$

Este concepto de "horizonte" fusiona varios motivos filosóficos tradicionales, tales como el concepto fenomenológico de "mundo" que desarrollaron casi contemporáneamente Husserl en La crisis de las ciencias europeas y Heidegger en Ser y tiempo. Por esa razón, que el hombre, Dasein o ego tenga mundo, por supuesto, no significa solamente que se mueva práctica y cognoscitivamente en un mundo o ambiente espacio-temporal de cosas, sino y antes que nada - que posea, proyectándolo sobre las cosas una y otra vez un horizonte interpretativo que pone esas cosas en una perspectiva determinada. El ser de las cosas, su comprensibilidad, su interpretabilidad, se fija a partir de la perspectiva que proporciona el horizonte, porque están interpretadas en vista a él. De esta forma, el horizonte, que consiste en un conjunto de sentidos interconectados, a veces explícitos, a veces implícitos, es la condición de la comprensibilidad de las cosas del mundo humano, que el hombre descubre en sus múltiples formas de interacción con ellas. En este sentido, poseer un mundo es, literalmente, poseer un horizonte comprensivo e interpretativo que determina nuestro acceso a las cosas "mundanas". Además, dado que, en el fondo, poseer un horizonte es poseer un lenguaje, es este último el que nos garantiza la posibilidad de un tal acceso a las cosas, de expresarlas y de 


\section{Dossiê Leibniz, Dissertatio - Volume Suplementar 03 I UFPel [2016]}

comunicarlas. $^{5}$ No por nada uno de los enunciados fundamentales de la hermenéutica gadameriana es "el ser que puede ser comprendido es lenguaje". ${ }^{6}$

Por otro lado, así como el horizonte nos brinda una perspectiva en relación con la cual ponemos las cosas, a la manera de un punto de fuga, así también el horizonte mismo es concebido como un aspecto según el cual interpretamos o comprendemos el mundo, en el sentido de que, de manera más bien oculta, nuestros horizontes particulares se nos presentan como un cierto aspecto de la totalidad: es el todo el que se nos presenta de una manera horizóntica: toda comprensión e interpretación, lo mismo que todo lenguaje, aspira a la universalidad, a poder enunciar, mediante sus recursos, todo lo que puede ser dicho. Ciertamente, cada horizonte de comprensión es una posibilidad del mundo, aunque única en su peculiaridad. Así, el rasgo fundamental del mundo humano es que siempre se presenta bajo la forma de horizontes aspectuales o respectivos. ${ }^{7}$ El "mundo horizóntico" es una perspectiva de algo que se nos presenta y se nos exhibe en escorzo, de modo tal que nunca podrá dársenos en toda su plenitud, del mismo modo que, cuando contemplamos un paisaje, no podemos hacerlo en forma simultánea desde todas sus perspectivas. Así, el mundo de las cosas y de nosotros mismos siempre se presentará bajo la condición de un horizonte, es decir, de manera parcial y perspectivista. De esta forma, para la razón finita no hay un más allá del horizonte en el que idealmente 
pudiese totalizarse, en una captación sin fisuras, el ser de las cosas mundanas. En efecto, el ser mismo de las cosas está dado por sus "posibilidades horizónticas", de forma tal que la razón humana, comprendida hermenéuticamente como la capacidad que tenemos de comprender e interpretar, es también de carácter perspectivista.

Esta forma de entender el perspectivismo, como un darse las cosas y el mundo bajo la forma de "respectos", se opone a una forma subjetivista de perspectivismo tal como, por ejemplo, se da en la interpretación heideggeriana de Nietzsche. En efecto, para Nietzsche el perspectivismo surge de la constricción de la vida humana a proyectar esquemas interpretativos para estabilizar el caos de la vida. Así, el horizonte, que es también correlación del concepto de perspectiva, constituye, para Nietzsche, una proyección del hombre, más que un aspecto o posibilidad de las cosas mismas. ${ }^{8}$ Como hemos dicho, quizá es por esta razón que la hermenéutica gadameriana no ha recogido plenamente la riqueza de las ideas leibnizianas, al menos como un momento de la hermenéutica. En efecto, Gadamer ve a Leibniz como un filósofo que está situado en un camino que lleva, finalmente, a esta concepción subjetivista (y hasta cierto punto irracional) del concepto subjetivista de perspectivismo. ${ }^{9}$

Un peligro que acecha a la hermenéutica es la imputación de sostener un relativismo absoluto. En efecto, podría alegarse que los diferentes horizontes 


\section{Dossiê Leibniz, Dissertatio - Volume Suplementar 03 I UFPel [2016]}

representan otras tantas pretensiones de "verdad", incompatibles entre sí e indecidibles. De esta forma, tendríamos así un relativismo que se expresa en términos de una inconmensurabilidad estricta: puede ocurrir que diferentes perspectivas del mundo capten aspectos de las cosas, pero ello no implica en sí mismo que sean integrables en una visión unificada de ellas. ¿No corremos el riesgo de que estos mutuos aspectos o perspectivas queden aislados unos de otros, como otros tantos mundos "monádicos"? La respuesta hermenéutica a esta severa objeción es su postulado de que los horizontes y las perspectivas que ellos implican son progresivamente integrables o fusionables en mundos aspectuales cada vez más abarcadores. A esta integrabilidad de los horizontes la denomina Gadamer "fusión de horizontes", cuya realidad actual en cuanto tal se cumple en la ejecución misma del acto de comprensión e interpretación. ${ }^{10}$ Sin embargo, la fusión de horizontes no es algo que la hermenéutica pueda justificar teóricamente, sino que, en todo caso, es para ella una condición cuya realidad se evidencia de manera fáctica y práctica en el hecho de que, finalmente, de una manera u otra, siempre comprendemos, interpretando e interpretamos, comprendiendo, a través de nuestro uso del lenguaje. ${ }^{11}$ En este sentido, el paradigma fundamental de la hermenéutica es la traducción de una lengua a otra, a través de la cual se nos revela que siempre hay un fondo común que permite pasar de un mundo de sentidos mediados lingüísticamente a otros. ${ }^{12}$ De esta 
forma, si aceptamos el concepto de fusión de horizontes y su optimismo lingüístico, resulta claro que, para la hermenéutica, la verdad, que consistiría en la posesión de un punto de vista final, conclusivo y verdaderamente universal acerca de las cosas, no está en el comienzo, sino al final, cuando hayan sido integrados y fusionados todos los horizontes de comprensión posibles en un único horizonte universal. Pero este fin final se revela como una tarea esencialmente inacabable, como un punto de fuga que, en la perspectiva, se ubica en el infinito. Esta tarea permanente en vista de una universalidad ideal requiere del lenguaje como medio universal y por ello, el diálogo y la dialéctica ocupan un papel fundamental en esa tarea infinita que se expresa, en la práctica, como búsqueda de acuerdos o entendimientos cada vez más amplios. ${ }^{13}$ Todo ello depende del postulado optimista de que hay una racionalidad dialógica o “dialéctica" y que el acuerdo, aún cuando sea provisorio, siempre es posible. Por esa motivo, la razón hermenéutica es más una práctica que un contenido final.

Ahora bien, esta nueva forma de optimismo racionalista mitigado depende de una condición ontológica de índole trascendental, si se quiere. Y es que el acuerdo dialógico es posible, al menos como ideal regulativo, porque en el fondo los diferentes aspectos horizónticos son siempre expresiones de lo mismo, que, en cuanto tal, aparece o se exhibe siempre de manera parcial. En el fondo, es el ser mismo de las cosas el que, mediante la acción humana, libera o despeja sus posibilidades interpretativas, y es ese mismo ser, incluyendo el de

${ }^{13}$ Gadamer (1960), p.375-384, esp. 383 [439-458, esp. 457]. 


\section{Dossiê Leibniz, Dissertatio - Volume Suplementar 03 I UFPel [2016]}

nosotros mismos, el que postulamos como aquello en que fundamos nuestra confianza en una unidad final.

Como he dicho anteriormente, hay una multiplicidad de tesis de la metafísica leibniziana que pueden rescatarse para la hermenéutica y, como intentaré indicar hacia el final, hallamos varios respectos en los que Leibniz puede ayudar a completar algunos territorios que no quedan cubiertos en la cartografía hermenéutica.

\section{El perspectivismo de Leibniz}

El punto de partida para poder establecer las conexiones entre la hermenéutica y la filosofía leibniziana es la consideración de la mónada como un ente que tiene mundo. En efecto, pareciera ser que la tesis leibniziana de que a la mónada nada le viene de fuera, sino que todo surge de "su propio fondo" y de que, por tanto, "la mónada no tiene ventanas", se encuentra en abierta contradicción con una de las ideas centrales de la hermenéutica, a saber, la apertura del ente comprensor, el Dasein, hacia el mundo. No obstante, a la concepción de la sustancia monadológica viene a añadírsele un aspecto significativo, el hecho de que toda mónada constituye, en cuanto tal y en su propio ser, un punto de vista, una perspectiva del mundo. De entre innumerables pasajes, citamos el famoso párrafo del Discurso de metafísica: 
producir para manifestar su gloria y mira todos los aspectos del mundo de todas las maneras posibles, puesto que no existe relación alguna que escape a su omnisciencia; el resultado de cada visión del universo, en el que éste comparece como contemplado desde un determinado lugar, es una sustancia que expresa el universo conforme a esa visión, con tal que Dios considere conveniente hacer efectivo su pensamiento y producir esa sustancia. ${ }^{14}$

Más allá de las razones por las que Leibniz llegó a esta concepción de la sustancia "real", el ser activo dotado de percepción y apetición, y si hacemos abstracción de ellas para quedarnos con algunos aspectos de la tesis central, resulta ser que la mónada leibniziana proporciona un modelo anticipado del modo de existencia fáctica que se encuentra en la base de la hermenéutica, a saber, el Dasein y, en la fenomenología, del ego como polo intencional. Este hecho es fácilmente comprobable, especialmente en el caso de Husserl, quien recurre al concepto de mónada o ego monádico en sus Meditaciones cartesianas. ${ }^{15}$ La mónada tiene "mundo", que es, precisamente, la cuestión central de la perspectiva, el punto de vista y del horizonte.

Heidegger ignoró este potencial hermenéutico de Leibniz, aunque hasta cierto punto quedó registrado en sus seminarios, por ejemplo, en los Metaphysische Anfangsgründe der Logik. ${ }^{16}$ Sin embargo, predominó en Heidegger una interpretación de Leibniz según la cual se encuentra en el origen del subjetivismo moderno y del predominio del principio de razón suficiente como

${ }^{14}$ A VI 4 1549-1550 [OFC 5 175-176].

${ }^{15}$ Husserl (1979), pp.121-122; 169; 173, inter alia.

${ }^{16}$ GA 26, pp.118-122. 


\section{Dossiê Leibniz, Dissertatio - Volume Suplementar 03 I UFPel [2016]}

expresión de la razón calculante, como hemos señalado al comienzo. Ya desde sus primeras interpretaciones de Leibniz, Heidegger lo aborda desde el punto de vista de la "fundamentación metafísica de la lógica" y ve en la mónada de Leibniz una interpretación del ser como pulsión (Drang). ${ }^{17}$ Probablemente, esta interpretación más bien negativa del autor de la Monadología haya influido también en la actitud general de Gadamer hacia la filosofía Leibniziana, a pesar de las esporádicas referencias positivas sobre Leibniz que encontramos en sus obras.

Naturalmente, hay cuestiones de la monadología que se conectan estrechamente con el subjetivismo y el solipsismo, en particular, no podemos dejar de mencionar la clausura del sujeto monadológico, que se expresa en la ya citada metáfora de la mónada sin ventanas. Ciertamente, es el tributo que paga Leibniz por su pertenencia al mundo moderno, expresado en el ego cogito cartesiano. Pero quizá encontremos en Leibniz mismo una salida a las aporías de una filosofía de la conciencia. En efecto, la respuesta leibniziana al problema del subjetivismo moderno está, precisamente, en la idea de la armonía universal como tesis metafísica fundamental, un aspecto de la filosofía leibniziana que ha sido desatendido o despreciado por Heidegger y, en consecuencia, por la hermenéutica posterior.

${ }^{17} \mathrm{GA} 26, \mathrm{pp} .111$. 
Como dice Leibniz, ser es "ser armónico" 18 y por ello, la trascendencia del sujeto monádico se funda, precisamente, en el ideal de la armonía y en la teoría de la armonía preestablecida en la que se funda el acuerdo universal de todos los entes que constituyen el mundo. Mientras que Heidegger puso el acento antes que nada en el Drang, en la pulsión como un elemento del sujeto, la apetición, despreció la tesis de la una unidad armónica. ${ }^{19}$ En efecto, La armonía universal es una tesis ontológica en el sentido estricto de la palabra: constituye una garantía que permite la comunicación universal entre todas las cosas. En todo caso, podemos concederle la jerarquía de un postulado o, si se quiere, de una fe filosófica. Desde el punto de nuestra existencia fáctica, es más que nada un ideal regulativo, de acuerdo con el cual tenemos que ajustar nuestros comportamientos respecto del mundo y de los demás. En este sentido, nuestras vidas se despliegan como si, efectivamente, las mónadas tuviesen ventanas. Si todo está ya antes coordinado entre sí, si hay una comunidad de todo con todo, podemos tener confianza en que no sólo en el mundo hay orden, sino también en que la aparente diversidad y contrastación de los puntos de vista, finalmente, tenderán hacia un acuerdo final.

Por esa razón, la armonía universal no sólo tiene un valor explicativo desde el punto de vista metafísico, en el sentido de que proporciona una explicación de cuestiones fácticas tales como la relación entre el alma y el cuerpo,

${ }^{18}$ AA VI 3, p.56; AA VI 3, p.474.

${ }^{19}$ Este aspecto está registrado en GA 26, pp.119-120, pero en Heidegger (2000b), p.90, Leibniz aparece en el camino que lleva hacia Nietzsche. 


\section{Dossiê Leibniz, Dissertatio - Volume Suplementar 03 I UFPel [2016]}

la concordancia del comportamiento dinámico de los cuerpos, o, finalmente, de la armonía de los estados perceptivos de todas las sustancias del mundo entre sí. Más allá de ello, la importancia para una perspectiva hermenéutica radica en que posee un valor decisivo para la vida práctica y, más aún, para el ejercicio de una cierta clase de actitud filosófica, puesto implica una concepción de la racionalidad humana. Si el ser es armónico, la razón humana puede siempre anticipar esa armonía y apuntar hacia ella, en el sentido de que el acuerdo, la concordancia, siempre es posible. Así, La armonía universal es la forma en que la razón finita se trasciende a sí misma y apunta, de manera contrafáctica, a la totalidad, de acuerdo con el lema "no quizá ahora, pero a la larga...". De esta forma, el mundo del sujeto monadológico no es solamente su mundo, sino también el de los otros y, en ese sentido, podemos decir que la mónada está abierta y que es, ella misma, una ventana. Dicho de otro modo, estamos hechos para el acuerdo y, por tanto, tenemos que tener confianza en que lo lograremos, a pesar de la diversidad de creencias y concepciones.

La armonía universal se expresa, fundamentalmente, en el hecho de que cada posición, cada postura filosófica, cada opinión contiene, de algún modo, la verdad. Así, hay un patrimonio de verdades que pasa de época en época y que se despliega al ritmo de su evolución. Es el ideal leibniziano de la perennis Philosophia, tal como se lo expresa a Remond, en la famosa carta del 27 de agosto de 1714 .

La verdad está más extendida de lo que se piensa, pero frecuentemente se encuentra encubierta y, más frecuentemente aún, envuelta e incluso 
debilitada, mutilada, corrompida por añadidos que la arruinan o la hacen menos útil. Haciendo ver estas huellas de la verdad en los antiguos o (para hablar con mayor generalidad) en los que nos precedieron, se extraería el oro del fango, el diamante de su mina y la luz de las tinieblas; y, en efecto, una cosa así sería perennis quaedam Pbilosopbia.

Se puede también decir que se puede comprobar un progreso en los conocimientos. Los orientales han tenido bellas y grandes ideas acerca de la Divinidad; los griegos le han añadido el razonamiento y una forma de ciencia. Los Padres de la Iglesia han rechazado lo que había de malo en la filosofía de los griegos. Mas los escolásticos han tratado de emplear con utilidad para el Cristianismo lo que había de aceptable en la filosofía de los paganos. Con frecuencia he dicho: aurum latere in stercore illo scholastico babariei. Y, personalmente, desearía que se pudiera encontrar un hombre hábil, versado en la filosofía irlandesa y española y que tuviese la capacidad de extraer todo lo bueno de ellas. ${ }^{20}$

No es importante para nuestro argumento el hecho de que, como todo filósofo, Leibniz se adjudique la tarea de conciliar todos los puntos de vista y extraer, finalmente, el oro oculto entre la escoria. Lo que importa para el punto de vista hermenéutico es que la verdad ya estaba allí, y es el intento de conciliación lo que la extrae de la parcialidad y el ocultamiento. La verdad surge de la conciliación y de la armonización de los puntos de vista, que tiene como efecto recoger lo común y eliminar la unilateralidad de los que persisten recalcitrantemente en su propia opinión. Al respecto, nada más claro que este otro pasaje, que proviene de las observaciones de Leibniz a Bayle. En efecto, al responder los comentarios de Bayle al Nuevo sistema de la naturaleza, Leibniz concluye:

La consideración de este sistema hace ver también que cuando se llega al fondo de las cosas, se observa mucha más razón de lo que se creía que se

${ }^{20} \mathrm{GP}$ III, pp.624-625. 


\section{Dossiê Leibniz, Dissertatio - Volume Suplementar 03 U UFPel [2016]}

podía encontrar en la mayor parte de las sectas de los filósofos: la poca realidad sustancial de las cosas sensibles de los escépticos, la reducción de todo a la armonía o números, ideas y percepciones de los pitagóricos y platónicos, lo uno y también el todo de Parménides y de Plotino, sin espinozismo alguno, la conexión estoica, compatible con la espontaneidad de los otros, la filosofía vital de los cabalistas y de los herméticos, que introducen sensaciones por todas partes, las formas y entelequias de Aristóteles y de los escolásticos y, a pesar de ello, la explicación mecánica de todos los fenómenos particulares según Demócrito y los modernos, y así a continuación, se encuentran reunidas como en un centro de perspectiva, desde donde el objeto (embrollado cuando se lo contempla desde otro lugar) deja ver su regularidad y la conveniencia de sus parte: se ha fallado sobre todo por el espíritu de secta, limitándose a rechazar a todos los otros. ${ }^{21}$

Así, los puntos de vista parciales aciertan por lo que afirman y se equivocan por lo que niegan o rechazan, aunque en el fondo son conciliables, si se adopta el punto de vista, la perspectiva correcta, en la que se recoge, en una mirada final, lo que hay de universalidad en todos ellos. Se podría afirmar que Leibniz mismo, en su práctica filosófica, lleva a cabo este ideal programático, no sólo porque con frecuencia escoge, como Platón, la forma de exposición dialógica, sino porque su misma filosofía se desarrolla en forma de controversia, a través del intercambio epistolar o, incluso, a través de la conversación personal, de la que, ocasionalmente, queda algún registro. No necesitamos referirnos a la importancia que ha tenido y tiene hoy en día la correspondencia leibniziana para la comprensión de su pensamiento, así como su necesidad perenne de hallar interlocutores con los que pudiese discutir sus concepciones. A la luz de esta necesidad imperiosa de diálogo se entiende también esa tendencia leibniziana, a

${ }^{21}$ GP IV, pp.523-524. 
veces patológica, de expresarse en los términos de su corresponsal o compañero de discusión..$^{22}$ No se trata de un mero artilugio retórico, como en ocasiones se ha sostenido, sino de una auténtica actitud hermenéutica de asumir el punto de vista del otro.

Por su parte, la armonía universal y el perspectivismo se conectan profundamente con el concepto de expresión, que constituye una de las claves fundamentales de la filosofía leibniziana. ${ }^{23}$ Precisamente, las consecuencias del concepto leibniziano de expresión han pasado inadvertidas para las interpretaciones subjetivistas de la filosofía monadológica. En efecto, la mónada no es únicamente pulsión o apetito, no se limita a re-presentar el mundo en el sentido de una mera imposición, sino que, al contrario, más bien lo expresa; dicho de otro modo, el punto de vista no es sólo un centro desde donde se mira, sino que es un punto desde donde algo aparece o se muestra. Dicho de otro modo, constituye un centro de concentración a la manera de un foco. El perspectivismo de Leibniz no es ni puramente subjetivo ni puramente objetivo, sino ambos al mismo tiempo y, por tanto, seguramente algo que posiblemente no pueda medirse únicamente mediante esa oposición polar. Del propio Leibniz hallamos una apreciación de la importancia de la expresión, en su respuesta a de Volder:

Parece que has examinado muy bellamente mi doctrina acerca del modo en
que cualquier cuerpo expresa todos los otros [cuerpos] y también el modo
en que cualquier alma o entelequia expresa no sólo su propio cuerpo, sino
también todos los otros a través de él. Pues bien, cuando hayas sopesado

22 Para el aspecto controversial y dialógico de la obra de Leibniz, ver Dascal (2008); De Olaso (1976); De Olaso (1990).

${ }^{23}$ Esquisabel (2016), pp.73-87. 


\section{Dossiê Leibniz, Dissertatio - Volume Suplementar 03 I UFPel [2016]}

cuidadosamente su alcance, verás que no hay nada que yo haya sostenido que no sea consecuencia de ella. ${ }^{24}$

Ya hemos dicho anteriormente que hay dos formas básicas de perspectivismo, el subjetivista, tal como el que podemos hallar en Nietzsche, para el que hay una proyección de horizontes desde el sujeto hacia el mundo, y otro objetivista, de acuerdo con el cual el mundo y las cosas mundanas se nos presentan en un cierto escorzo, en una cierta perspectiva, en el marco de un horizonte determinado. Hemos tratado de mostrar que la hermenéutica responde más bien al segundo modelo, antes que al primero: los aspectos u horizontes no son meras proyecciones del sujeto, sino que son modos de presentación del ser mismo de las cosas.

El perspectivismo de Leibniz es más bien de este último tipo, aunque, como vimos, por su énfasis en la noción de sujeto, comparte aspectos del primero. En esencia, podríamos hablar de un perspectivismo expresivista o también de un representacionismo expresivista. Para comprender esta forma de representacionismo, hay que tener en cuenta las fuentes geométricas del concepto de perspectiva y de expresión. En efecto, en el concepto leibniziano de punto de vista y de perspectiva hay una notable influencia de los desarrollos de la geometría proyectiva del siglo XVII, cuyos representantes más conspicuos fueron Desargues y Pascal. Este hecho ha sido señalado ya por M. Serres ${ }^{25}$ y, 
más recientemente, por Debuiche. ${ }^{26}$ En Desargues encontramos un conjunto de conceptos e instrumentos que innovaron el tratamiento de los problemas de las secciones cónicas y de perspectiva mediante la introducción de conceptos "ideales", tales como puntos situados en el infinito. Leibniz no cita frecuentemente a Desargues, pero es clara su influencia en él. ${ }^{27}$ Por su parte, Pascal expuso sus famosos teoremas sobre las propiedades proyectivas de las cónicas, gracias a las cuales se dan entre ellas transformaciones recíprocas. ${ }^{28}$ Estos desarrollos de las geometrías proyectivas le procuraron a Leibniz la base para sostener una teoría perspectivista fundada en la equivalencia de los puntos de vista. En efecto, una de las características de la geometría proyectiva es que en general, una proyección realizada desde un centro de proyección puede ser transformada en otra equivalente, realizada desde otro centro de proyección. Teóricamente, esta situación se debe a que, en general, se trata de transformaciones lineales que tienen como propiedad fundamental el isomorfismo estructural. Desde este punto de vista, se puede hablar de invariantes bajo transformaciones, lo cual significa que la posibilidad de transformaciones isomórficas se basan en el hecho de que a todas las proyecciones geométricas le subyace una misma estructura. ${ }^{29}$ Esta concepción, que se encuentra presente en Leibniz y que anticipa de manera larvada la teoría

\footnotetext{
${ }^{26}$ Debuiche (2009), pp.88-117.

${ }^{27}$ Debuiche (2009), pp.100-102

${ }^{28}$ Debuiche (2009), ibídem.

${ }^{29}$ Debuiche (2009), pp.102-104; Esquisabel (2016), pp.84-87. Para la cuestión de la identidad estructural, cfr. Esquisabel (2008).
} 


\section{Dossiê Leibniz, Dissertatio - Volume Suplementar 03 I UFPel [2016]}

contemporánea de los morfismos, constituye uno de los pilares de su concepto de expresión y también de su teoría de la representación. En definitiva, la expresión puede entenderse como una universalización de propiedades que subyacen a los teoremas de la geometría proyectiva. Así, se la puede concebir como una relación estructural que permite transformar un punto de vista en otro, sobre la base de una identidad estructural. De esta forma, las propiedades proyectivas garantizan que haya no sólo una correspondencia entre objeto y representación, sino también entre las diferentes perspectivas de las que resultan diferentes proyecciones de un mismo objeto. De esta forma, Leibniz asume una de las consecuencias de una concepción proyectiva de los objetos: todas las proyecciones de un mismo objeto son transformables entre sí mediante un conjunto de operaciones de transformación. Por esa razón, Leibniz sostiene que los diferentes puntos de vista no sólo expresan o representan lo mismo, sino que se interexpresan o representan entre sí: cada punto de vista es transformable en todos los restantes, dado que cada uno de ellos es una proyección de un mismo mundo o realidad. Al respecto, un notable texto de la Théodicée, el parágrafo 357 sintetiza esta idea de una manera clásica:

Es verdad que la misma cosa puede ser representada de maneras diversas, pero debe haber siempre una relación exacta entre la representación y la cosa y, en consecuencia, entre las diferentes representaciones de una misma cosa. Las proyecciones de perspectiva, que resultan en el círculo en las secciones cónicas, hacer ver que un mismo círculo puede ser representado por una elipse, por una parábola y por una hipérbola e incluso por algún otro círculo, por una línea recta o por un punto. Nada parece tan diferente ni tan desemejante que estas figuras. $\mathrm{Y}$, sin embargo, existe una relación exacta de cada punto con cada punto. Del mismo modo, hay que reconocer 
que cada alma se representa el universo según su punto de vista y por una relación que le es propia. Pero una perfecta armonía subsiste en ella siempre. ${ }^{30}$

De esta forma, la expresión garantiza tanto el carácter representacional del punto de vista, como la posibilidad de establecer una relación armónica entre diferentes puntos de vista, a pesar de que parezcan diferentes. Por decirlo de alguna manera, la objetividad está dada por la posibilidad de traducir o transformar una perspectiva en otra: lo que las une es, en el fondo, una estructura común. Así, el objeto, como aquello que garantiza la comunidad de la estructura, es accesible a través de "perspectivas". Es precisamente esa "comunidad de estructura" la que, en el límite, garantiza la posibilidad de transformar los mundos "monádicos" entre sí. Por consiguiente, la concepción leibniziana de la representación va más allá de la mera función sustitucional o de la mera "posición" subjetivizante: en efecto, representar es expresar y, por esa razón, podemos hablar de un representacionismo "expresivo".

La vía de la armonía universal nos ha conducido al concepto de expresión. Así, la expresividad se encuentra en la base de la armonía y, por tanto, de la integrabilidad de las perspectivas y puntos de vista: se trata de diferentes accesos o aproximaciones a "lo mismo". Por eso me atrevo a concluir que, para Leibniz, ser armónico es ser expresivo. Por otra parte, en la medida en que las relaciones de semejanza formal constituyen el tejido de la filosofía leibniziana 


\section{Dossiê Leibniz, Dissertatio - Volume Suplementar 03 I UFPel [2016]}

en la que se cimenta la noción misma de expresión, la universalidad de la relación de expresión, entendida desde este punto de vista estructural, se hace presente en todos los planos del pensamiento de Leibniz, en la física, en la dinámica, en su antropología y también en sus concepciones acerca de la lógica, la matemática y, en particular, en su concepción del conocimiento simbólico, como si se tratase de un entramado de vasos comunicantes.

En conclusión, es en la expresión que encontramos el correlato leibniziano de la fusión de horizontes gadameriana, de la que es una versión “estructural", precisamente porque constituye una garantía de la integrabilidad de los diferentes puntos de vista, en una visión progresivamente común. Si ello es así, aunque los mundos monádicos parezcam estar clausurados, no lo están en realidad. Ciertamente, podemos decir que la clausura es sólo causal, pero ello no implica que no haya un tipo de conexión más elevada y anterior, que está dada, precisamente, por la expresión, que constituye, en el fondo, una relación de relaciones, una relación entre formas. Ciertamente, podría darse toda una axiomática de la relación de expresión como relación formal, entre cuyas propiedades se encuentra, claramente, la transitividad, como lo muestra claramente el texto citado. De hecho, la teoría de los morfismos y su generalización en la actual teoría de las categorías podrían concebirse, precisamente, como esa "axiomática" de la teoría de la expresión.

No obstante, si el modelo geométrico le presta a la teoría de la expresión un armazón formal, no por ello se agota en los aspectos meramente 
estructurales. Ciertamente, el morfismo estructural es sólo un modelo (una "representación”) al que apela Leibniz para clarificar un concepto mucho más complejo, por su dimensión metafísica y ontológica. En efecto, la expresión obedece a un dinamismo metafísico que se cumple en un movimiento que va de la inmanentización y concentración a la extrinsecación y dispersión. Dicho de otro modo, la mónada, el individuo sustancial, resulta de un movimiento de interiorización del mundo, en la medida en que es la concentración de una multiplicidad en una unidad. Al mismo tiempo, lo expresado, el mundo, que es conservado en la concentración de lo expresante y permanece en él, se despliega a través de los estados del expresante, que, mediante el movimiento de la memoria, la percepción y la apetición, desarrolla ese punto de vista que ya era, desde siempre, el expresante mismo. La mónada "tiene mundo" porque encuentra en sí misma las condiciones de su propia trascendencia, que se cumple siempre en este doble movimiento de concentración y expansión. Y si es un espejo del universo, no lo es porque lo refleje en una imagen anamórfica, sino porque ella misma es ya, en cierta forma, ese mundo. En esa trascendencia “inmanente" que es cada mónada, ella sale de sí misma para retornar en cierta forma, a sí misma. La expresión, metafísicamente considerada, no es ni más ni menos que este movimiento de interiorización, concentración y conservación en una exteriorización que al mismo tiempo desarrolla y dispersa. Dicho de otra manera, lo que es expresado está siempre en el que expresa y se conserva en él. 


\section{Dossiê Leibniz, Dissertatio - Volume Suplementar 03 I UFPel [2016]}

Quizá esta breve reflexión metafísica sobre la expresión nos proporcione una perspectiva nueva acerca de la relación entre el conocimiento claro y distinto, por un lado, y el confuso y oscuro, por el otro. En efecto, la comprensión del mundo surge de aquellos aspectos que le son inaccesibles al sujeto, pero que ya expresan el mundo en perspectiva: todo le nace al alma de su propio fondo o, dicho de otro modo, la contingencia del sujeto ya contiene sus posibilidades comprensivas, aunque al sujeto mismo no le sean, ni le puedan ser, completamente transparentes: la autorreflexión y la autoaclaración del sujeto no es sino una mínima parte de una tarea que se presenta, en cierto modo, infinita. De este modo, como para la hermenéutica, también para Leibniz hay una opacidad del sujeto monádico, que, lo mismo que para la hermenéutica, está ligada a su contingencia. Y, lo mismo que para la hermenéutica, así como el conocimiento del mundo es, en cierta forma, una manera de conocerse a sí mismo, la tarea del sujeto leibniziano es también precisamente eso: una tarea infinita a través de lo oscuro y confuso hacia lo claro y lo distinto, por todos sus grados.

Tres cuestiones, para finalizar estas consideraciones, querría añadir como motivos de reflexión hermenéutica en Leibniz. En primer lugar, desearía presentar una breve consideración hermenéutica con referencia a la cuestión de los mundos posibles. Ciertamente, los mundos de Leibniz son mundos ónticos (o "posibilidades fácticas", en el sentido un conjunto de cosas "posiblemente contingentes"), pero tampoco debe escapársenos su potencial hermenéutico en 
el sentido de que son también "otras posibilidades de ser", incluso extraordinariamente ajenas a las formas factuales en que se da nuestro mundo actual. O dicho en otra forma, estos "mundos posibles" no son meramente variaciones combinatorias del nuestro; por el contrario, puede y debe haber mundos posibles que no tienen nada en común con el que actualmente habitamos. Recordemos que la mística pirámide del final de la Théodicée tiene una base infinita. ${ }^{31} \mathrm{Y}$ si especulamos un poco más y recordamos que la expresión es transitiva, podríamos concluir entonces que, a pesar del carácter completamente ajeno de esos otros mundos, de un modo u otro tienen que estar expresados en este nuestro mundo y, por tanto, también tienen que haber dejado una huella en la constitución de nuestro propio ser contingente. Si ello es así, llevamos siempre en nosotros mismos las trazas de la alteridad de esos otros mundos.

La segunda cuestión colateral que deseo tocar, aunque más no sea que para dejar un registro de ella, compete a la inefabilidad de las cosas: individuum est ineffabile. Para la hermenéutica, a pesar de la infinita expansibilidad de nuestros horizontes de comprensión, las cosas definitivamente se nos escapan, no hay una ratio ultima para ellas, porque, en el fondo, siempre pueden dar más de sí, es decir, contienen una "reserva de sentido". Podemos preguntarnos, entonces, si en la concepción del análisis infinito de Leibniz, más allá de su fundamentación en la lógica del enunciado, no hay verdaderamente una anticipación de esta idea. En efecto, las cosas mundanas, contingentes, son inagotables para la razón

${ }^{31} \mathrm{GP}$ VI, p.364 [OFC 10, pp.370-371]. 


\section{Dossiê Leibniz, Dissertatio - Volume Suplementar 03 I UFPel [2016]}

humana, y cuando se embarca en su explicación, se abisma ante la necesidad de recorrer un mundo inabarcable e infinito, del que sólo puede tener, en el fondo, la certeza de que ha sido hecho de acuerdo con peso y medida, pero tal que ella misma no puede verificarlo por sí misma, por ser finita. En esta perspectiva, como lo ha indicado Jean Grondin, el principio de razón suficiente, entendido como el principio del análisis infinito, adquiere un matiz hermenéutico, que lo saca del quicio de la razón calculante: siempre se puede dar una razón ulterior, pero nunca la última, al menos dentro del ámbito de lo finito. ${ }^{32}$

Nuestra tercera consideración se vincula precisamente con el tema de la razón calculante. Frente a la visión pesimista de la hermenéutica relativa a este destino de la razón moderna, sostengo no sólo que las críticas dirigidas hacia Leibniz han sido injustas, sino también que nuestro filósofo exhibe cierta superioridad frente a la miopía de las posiciones hermenéuticas respecto de lo que podríamos denominar el "ser formal" de las cosas. En efecto, si hay una deficiencia de la hermenéutica es su relación con las ciencias, que parecen haber pasado a su lado sin haber dejado huella alguna. Quizá esto se deba al dictum heideggeriano de que la ciencia no piensa y que todo lo que tenga que ver con ella pertenece al ámbito de la tecnociencia, que finalmente conducirá al mundo a una forma imperceptible de aniquilamiento. De una manera u otra es una actitud que, aunque atenuada, podemos encontrar también en la hermenéutica gadameriana, en la forma de una condena del pensamiento científico en el

\footnotetext{
${ }^{32}$ Grondin (2000), p.6 (version on-line).
} 
sentido de una instrumentalización o cosificación del mundo y del hombre, en el sentido de una puesta a disposición de las cosas para el mero dominio por el dominio mismo. ${ }^{33}$ Precisamente, una de las pocas ocasiones en que Gadamer se refiere de manera más o menos extensa a Leibniz en Verdad y método acontece en forma de crítica del programa de la característica universal, que representa una degradación instrumentalizante y algorítmica de la capacidad poética del verdadero lenguaje. ${ }^{34}$ No obstante, en sus obras más tardías, Gadamer mismo reconoce que lo matemático, por ejemplo, ha sido un territorio inaccesible para la hermenéutica y que permanece frente a ella como un enigma indescifrable. ${ }^{35}$ Esta cuestión nos conduce al problema de la razón formal en Leibniz y a la discusión reciente acerca de la posibilidad de una "soft rationality" o racionalidad blanda. ${ }^{36}$ En todo caso, la degradación hermenéutica de la razón simbólica leibniziana al rango de razón calculante o mero instrumento simbólico de naturaleza algorítmica constituye una reducción injusta del papel que le asignó Leibniz a la construcción de lenguajes formales.

Es cierto que desde principios del siglo XX se ha asociado la filosofía leibniziana con el ideal de demostración rigurosa, en el que la lógica ocupa un lugar central. Pensemos, por ejemplo, en el ideal leibniziano de la demostración de los axiomas matemáticos mediante su reducción a identidades. Ciertamente,

\footnotetext{
${ }^{33}$ Gadamer (1960), pp.457-460 [543-547].

${ }^{34}$ Gadamer (1960), pp.418-421 [498-500].

35 Gadamer (1999), pp.362-363 [151-152].

${ }^{36}$ Blandior ratio, de acuerdo con la denominación de M. Dascal. Cfr. Dascal (s/f), http://www.tauc.ac.il/humanities/philos/dascal/papers/berlin.html
} 


\section{Dossiê Leibniz, Dissertatio - Volume Suplementar 03 I UFPel [2016]}

el énfasis puesto en las formas lógicas ha inspirado interpretaciones como las de Russell y Couturat, que han sido sumamente influyentes en la recepción del pensamiento leibniziano durante gran parte del siglo XX. ${ }^{37}$ No obstante, en la actualidad se rechazan estas interpretaciones clásicas, por ser unilaterales.

Ciertamente, no cabe duda de que se trata de una visión parcial, pero tampoco se puede negar que si Leibniz cumplió, por ejemplo, un papel inspirador en el programa logicista de Frege, es porque en su filosofía había elementos e ideas que se conectaban estrechamente con un programa de fundamentación de las ciencias fundado en la lógica. El problema no es que no haya ideas logicistas en Leibniz, sino más bien que sean las únicas que podamos encontrar en él. En todo caso, más allá de las disputas acerca de si hubo un programa fundacional leibniziano basado en el ideal de demostración fuerte, las reflexiones epistemológicas leibnizianas se destacan por la importancia que le otorgaron a la función del simbolismo y a la creación de sistemas semióticos al servicio del conocimiento. Precisamente, el concepto leibniziano de conocimiento simbólico sintetiza el carácter central de lo semiótico en la epistemología leibniziana. Al mismo tiempo, nos revela que en el programa leibniziano de crear sistemas de expresión adecuados a las ciencias no se trata justamente de una razón "algorítmica" o "calculante", sino que, antes bien, se trata de ganar mayor claridad y expresividad para las estructuras formales

\footnotetext{
${ }^{37}$ Russell (1900); Couturat (1901).
} 
involucradas en la articulación de nuestro conocimiento. ${ }^{38}$ Por esa razón, la invención de nuevas formas de representación conlleva una revolución simbólica. ${ }^{39}$ En particular, la formalización simbólica se pone al servicio de la invención y la creación matemática, no tanto porque se trate de una razón mecánica y algorítmica, sino porque un sistema de notación exacto y preciso proporciona un acceso más directo a lo matemático y lo lógico, que consisten, en lo fundamental, en estructuras, es decir, en plexos de conexiones y relaciones. En cierto modo, Leibniz supera así la limitación de la hermenéutica, en la medida en que, a través de la razón simbólica, reconoce un acceso peculiar a la dimensión formal, que es el ámbito propio de lo matemático y lo lógico. En este sentido, lo lógico y lo matemático constituyen también un "aspecto" de las cosas, cuyo modo de acceso es también de carácter perspectivista, en la medida en que se hace posible a través de diversos sistemas semióticos de carácter más o menos "formal".

En efecto, el conocimiento simbólico que obtenemos mediante sistemas semióticos especialmente diseñados para las ciencias es de carácter eminentemente estructural, en la medida en que la expresión simbólica (por ejemplo, una ecuación) muestra o "exhibe" la forma o estructura del objeto. De este modo, la representación estructural que nos proporciona la formación simbólica puede ser considerada también como "modo de presentación" de la 


\section{Dossiê Leibniz, Dissertatio - Volume Suplementar 03 I UFPel [2016]}

estructura. De esta manera, el carácter algorítmico o "calculante" es, en todo caso, un subproducto del programa de formalización simbólica. En suma, el objetivo central del desarrollo de sistemas simbólicos consiste en que podamos acceder de manera operativa a estructuras formales fundamentales, que están a la base de todo conocimiento.

En esta perspectiva, la idea leibniziana de lo "lógico" (es decir, de lo formal o estructural) en un sentido amplio está conectada no tanto con la posibilidad de hacer demostraciones apodícticas, apelando a mecanismos formales que garanten nuestras inferencias, sino más bien con el hecho de que hay relaciones estructurales que configuran y constituyen los diversos campos del saber y que, en el fondo, configuran una suerte de ser formal de las cosas, por lo que constituyen sus condiciones ontológicas. Por esa razón, podríamos decir que, desde un punto de vista contemporáneo, el racionalismo de Leibniz, o su optimismo racionalista, es más bien matemático-estructural, antes que lógico-formal. Sin embargo, que las estructuras sean condiciones del ser de las cosas no significa que para nosotros sean completamente accesibles y expresables. Sólo hay diversas aproximaciones y representaciones simbólicas de estas relaciones estructurales, sin que lleguemos a agotarlas de manera total. Dicho de otra manera, si las formas estructurales nos son accesibles a través de la complejidad de los sistemas simbólicos, no hay ni puede haber un único sistema de representación que clausure la investigación humana. Para parafrasear a Pascal, la estructura es una esfera cuyo centro está en todas partes 
y su circunferencia en ninguna. Para Leibniz, lo formal, lo estructural, se expresa en los diversos sistemas simbólicos de una manera parcial y perspectivista, con mayor o menor eficiencia y claridad, con mayor o menor perspicuidad..$^{40}$

En el fondo, no hay contradicción entre la razón formal o simbólica y la idea de una razón hermenéutica o "finita". Ciertamente, la razón formal opera con el ideal regulativo de que las formas o estructuras son las que están al comienzo de nuestra investigación, como condición de posibilidad, y al final, como una meta deseable, pero tal vez no completamente asequible. De esta manera, el formalismo es el instrumento que poseemos no tanto para demostrar como para investigar, en su riqueza, la inagotabilidad de las formas. Finalmente, la razón formal o simbólica es una forma de la razón, pero no la única. Las conexiones y relaciones que pueden ser formalizadas en un sistema simbólico constituyen una guía de la investigación, al tiempo que proporcionan instrumentos para organizar y aumentar nuestros conocimientos; sin embargo, la razón formal es sólo un aspecto de la racionalidad, justamente por limitarse al carácter "estructural" de las cosas.

\section{Conclusión}

He tratado de mostrar que se pueden señalar puntos de contacto entre la hermenéutica de cuño gadameriano y la filosofía leibniziana, a pesar de las diferencias que puedan señalarse y de la aproximación crítica la filosofía

${ }^{40} \mathrm{Cfr}$. Serres (19680, pp.534-554 


\section{Dossiê Leibniz, Dissertatio - Volume Suplementar 03 I UFPel [2016]}

hermenéutica pueda haber sostenido frente a ciertas tesis de Leibniz, como, por ejemplo, su posible pertenencia al subjetivismo moderno. He argumentado que tanto en Gadamer como en Leibniz se puede encontrar un perspectivismo no subjetivizante, en la medida en que, en ambos, el punto de vista no es una mera posición del mundo, sino más bien el acceso al mundo a partir de un aspecto que él mismo nos ofrece. Y así como existe un optimismo interpretativo, en la medida en que la integración de perspectivas se realiza mediante la fusión de horizontes, a través de formas dialógicas, así también el carácter expresivo de la razón finita está en la base de la posibilidad de integrar los puntos de vista parciales hacia una razón común, que ya estaba, oculta, desde el principio. Sin embargo, hay un punto en el que Leibniz aventaja a la hermenéutica, y es aquél en el que esta última, precisamente, es más crítica respecto del pensamiento leibniziano: la razón simbólica, lejos de ser una mera razón calculante y ciega, constituye una forma de acceso a aquél dominio que, hasta cierto punto, sigue siendo una terra incognita para la hermenéutica. En efecto, la razón, en la medida en que es esencialmente simbólica, accede al dominio de lo formal, que constituye también una dimensión del ser de las cosas y, en la misma medida en que sus otras dimensiones, se nos presenta siempre en escorzo, de manera perspectivista, a través de la multiplicidad de los sistemas semióticos, entre ellos, también los lenguajes históricos. 


\section{Ediciones de los escritos de Leibniz}

LEIBNIZ, G.W. (1923-). Sämtliche Schriften und Briefe. Herausgegeben von der Deutschen Akademie der Wissenschaften, Darmstadt, 1923; Leipzig, 1938; Berlin, 1950 y prosigue.

(1875-1890). Die philosophischen Schriften von Gottfried Wilhelm

Leibniz: GERHARDT, C. I. (hrsg), 7 Bände, Berlin. (2007-). Obras filosóficas y cientificas. Sociedad Española Leibniz

(ed.). NICOLÁS, J. (director). Granada: Editorial Comares.

\section{REFERENCIAS BIBLIOGRÁFICAS}

COUTURAT, Louis (1901). La logique de Leibniz après ses manuscrites inédites. Paris: Alcan.

DASCAL, M. (comp.) (2008). Leibniæ: What Kind of Rationalist?. Springer.

(s/f), "Nihil sine ratione: Blandior Ratio".

http://www.tauc.ac.il/humanities/philos/dascal/papers/berlin.html

DE OLASO, E. (1975). "Leibniz et l'art de disputer. En: Akten des II. Internationalen Leibniz-Kongresses, vol. 4 (=Studia Leibnitiana Supplementa 15). 


\section{Dossiê Leibniz, Dissertatio - Volume Suplementar 03 UFPel [2016]}

(1990). "Sobre la filosofía leibniziana de las controversias". En:

Gil, F. (ed.), Controvérsias Científicas e Filosóficas. Lisboa: Fragmentos.

DEBUICHE, Valérie. (2009). "La notion d'expression et ses origins mathématiques". Studia Leibnitiana, XLI, pp. 88-117.

ESQUISABEL, Oscar M. (2008). “Leibniz’s Science of Forms as a Structural Science and Similarity as its Central Concept". CLE e-Prints, vol 8, n 6. http://www.cle.unicamp.br/e-prints/vol_8,n_6,2008.html

(2012). "Representing and Abstracting. An Analysis

of Leibniz's Concept of Symbolic Knowledge”. En: Lassalle Casanave, Abel, Symbolic Knowledge from Leibniz to Husserl. London: College Publications, pp.1-49. (2016). "Perspectivism, Expression, and Logic in Leibniz. A Foundational Essay". En: Wenchao Li; Beckmann, Ute; Erdner, Sven; Errulat, Esther-Maria; Herbst, Jürgen; Iwasinski, Helena y Noreik, Simona (comps.), Vorträge des X. Internationalen Leibniz-Kongresses "Für unser Glück oder das Glück anderer". Hildesheim-Zürich-New York: Georg Olms, Band III, 73-87.

GADAMER, Hans-Georg (1960). Wabrheit und Methode. Grundzüge einer philosophischen Hermeneutik. Tübingen, J.C.B. Mohr. Ulteriores ediciones: 1965, 1972 (ampliada), 1975 (reimp. De 1972), 1986 (ampliada y revisada); 1990 (revisada): vol. 1 de Gesammelte Werke. Se cita por edición de 1990. [Trad. esp. Verdad y método I. Fundamentos de una bermenéutica filosófica, trad. de Ana Agud 
Aparicio y Rafael de Agapito, Salamanca, Sígueme, 1993 (5ta. Edición, según edición 1975)].

GADAMER, Hans-Georg (1990). "Festrede zum 300. Geburtstag von Gottfried Wilhelm Leibniz am 1. Juli 1946 in der Aula der Universität Leipzig”, Studia Leibnitiana, 22/1.

GADAMER, Hans-Georg, (1999). "Musik und Zeit. Ein philosophisches Postsriptum", en: Ästhetik und Poetik. I. Kunst als Aussage, Tübingen, Mohr Siebeck (GA, volume 8), pp. 362-363 [“'La música y el tiempo. Un postscriptum filosófico", en: Arte y verdad de la palabra, trad. José Francisco Zúñiga García y Faustino Oncina, introd. Gerard Vilar, Barcelona, Paidós Ibérica, 1998, pp. 151152].

GRONDIN, Jean. (2000) “Das Leibnizsche Moment in der Hermeneutik”. En: Beetz, Manfred und Cacciatore, Giuseppe (eds.). Die Hermeneutik, in Zeitalter der Aufklärung, Köln/Weimar/Wien: Böhlau Verlag, pp. 3-16. Versión electrónica:http://mapageweb.umontreal.ca/grondinj/pdf/das_leibnizische_ moment.pdf

HEIDEGGER, Martin (1978). Metaphysische Anfangsgründe der Logik im Ausgang von Leibniæ, Frankfurt am Main. Vittorio Klostermann (GA, Band 26).

(2000ab) Nietssche I (2000a)-Nietzsche II (2000b).

VERMAL, trad. J L. (trad.). Barcelona: Ediciones Destino. 
HUSSERL, E. (1979), Meditaciones cartesianas. PRESAS, M. A. (introd., trad. y notaS). Madrid: Ediciones Paulinas.

RUSSELL, Bertrand (1900). A Critical Exposition of the Philosophy of Leibniz. London (2d. Ed. 1937) [citado por la edición de Routledge, 2005].

SERFATI, Michel, (2005). La revolution symbolique. La constitution de l'écriture symbolique mathématique. Paris: Pétra.

SERRES, Michel (1968). Le systeme de Leibniz et ses modèles mathématiques. Paris: PUF, 1968 [1982, 2a. ed.].

E-mail: omesqui1@speedy.com.ar 